\title{
Sevoflurane exposure in postnatal rats induced long-term cognitive impairment through upregulating caspase-3/cleaved-poly (ADP-ribose) polymerase pathway
}

\author{
YUNZHI LING $^{1 *}$, XIAOHONG LI ${ }^{1 *}$, LI YU $^{2}$, QISHENG LIANG $^{1}$, XUEWU LIN ${ }^{1}$, \\ XIAODI YANG ${ }^{3}$, HONGTAO WANG $^{4}$ and YE ZHANG $^{5}$
}

\begin{abstract}
${ }^{1}$ Department of Anesthesiology, First Affiliated Hospital of Bengbu Medical College, Anhui, Hefei 233004; Departments of ${ }^{2}$ Laboratory Medicine, ${ }^{3}$ Parasitology, and ${ }^{4}$ Immunology, Bengbu Medical College, Anhui, Hefei 233030; ${ }^{5}$ Department of Anesthesiology, Second Affiliated Hospital of Anhui Medical University, Anhui, Hefei 230601, P.R. China
\end{abstract}

Received September 8, 2016; Accepted May 16, 2017

DOI: 10.3892/etm.2017.5004

\begin{abstract}
The association of anesthetic exposure in infants or young children with the long-term impairment of neurologic functions has been reported previously; however, the underlying mechanisms remain largely unknown. In order to identify dysregulated gene expression underlying long-term cognitive impairment caused by sevoflurane exposure at the postnatal stage, the present study initially performed behavioral tests on adult Wistar rats, which received $3 \%$ sevoflurane at postnatal day 7 (P7) for different time course. Subsequently, transcriptome profiling of hippocampal tissues from experimental and control rats was performed. Significant impairment of the working memory was observed in adult rats with sevoflurane exposure for 4-6 h, when compared with the control rats. The results indicated that a total of 264 genes were aberrantly expressed (51 downregulated and 213 upregulated; fold change $>2.0 ; \mathrm{P}<0.05$; false discovery rate $<0.05$ ) in the hippocampus of experimental adult rats compared with those from control rats. Particularly, the expression of caspase- 3 gene (CASP3), encoding caspase-3 protein, presented the most significant upregulation, which was further validated by quantitative polymerase chain reaction and immunohistochemical analysis. Further analysis revealed that $C A S P 3$ expression
\end{abstract}

Correspondence to: $\mathrm{Dr} \mathrm{Li} \mathrm{Yu}$, Department of Laboratory Medicine, Bengbu Medical College, 2600 East Sea Road, Anhui, Hefei 233030, P.R. China

E-mail: yuli95@163.com

Dr Ye Zhang, Department of Anesthesiology, Second Affiliated Hospital of Anhui Medical University, 678 Furong Road, Anhui, Hefei 230601, P.R. China

E-mail: zhang_ye011@163.com

*Contributed equally

Key words: sevoflurane, long-term cognitive impairment, caspase-3, cleaved-poly (ADP-ribose) polymerase level was negatively correlated with the rats' spatial working memory performance, as indicated by the Y-maze test. The level of cleaved-poly (ADP-ribose) polymerase (PARP), a substrate of caspase-3, was also increased in the hippocampus of experimental adult rats. Thus, the present study revealed that upregulation of caspase-3/cleaved-PARP may be involved in long-term cognitive impairment caused by sevoflurane exposure in infants, which may be useful for the clinical prevention of cognitive impairment.

\section{Introduction}

Although anesthesia can protect patients undergoing surgical procedures from significant pain, recent studies have demonstrated that exposure to inhaled anesthetics, such as sevoflurane and isoflurane, induced neuropathological alterations in experimental animals $(1,2)$. In addition, several lines of evidence from animal models have suggested that excessive exposure to volatile anesthetics may cause irreversible and long-term behavioral changes resembling autism spectrum disorders (3). In clinical practice, sevoflurane is the most widely used inhaled anesthetics, particularly for cesarean section and surgical procedures in infants and young children (4,5); thus, the present study focused on sevoflurane.

In humans, the association of anesthetic exposure in infants or young children with the long-term impairment of neurologic functions has been reported in several retrospective clinical studies. For instance, Kalkman et al (6) observed that children with exposure to anesthetic agents at an age $<24$ months presented more behavioral disturbances in comparison with those who were exposed to anesthetics after the age of 2 years. Similarly, Wilder et al (7) demonstrated that children receiving two or more types of anesthetics at the same time were at an increased risk of developing learning disabilities, with a hazard ratio of 1.59 , subsequent to retrospectively analyzing a cohort of 5,357 children.

To date, numerous dysregulated biological processes have been identified for underpinning the pathologic basis of neurologic function impairment caused by sevoflurane exposure. For instance, Zhang et al (8) reported that inhalation of 
sevoflurane may reduce synaptotagmin-1 protein levels in the hippocampus, which then reduced the efficiency of synaptic transmission, thus resulting in memory impairment in rats. In one of our previous studies, decreased PSD95 expression in the medial prefrontal cortex was also observed in mice with cognitive impairment induced by sevoflurane (9). Furthermore, Xiong et al (10) reported that the CREB signaling pathway was inhibited in aged rats following exposure to sevoflurane. However, it is unknown whether these dysregulated biological processes are implicated in sevoflurane-induced long-term neurologic functional impairment. Systematic investigation of the aberrantly expressed genes in animals exhibiting neurological dysfunctions due to exposure to sevoflurane at postnatal age is also lacking.

The hippocampus is a major component of the central nervous system, which is located inside the medial temporal lobe. The hippocampus structure contains two main parts, namely Ammon's horn and the dentate gyrus, as well as four relatively independent parts, including cornu ammonis 1 (CA1), CA2, CA3 and CA4. The hippocampus serves a crucial role in regulating the cognitive function (11). A previous study demonstrated that the hippocampus is vulnerable to adverse events, including hypoxemia, exposure to inhaled anesthetics and surgical trauma (12). Evidence from magnetic resonance imaging studies indicated that decreased hippocampal volume is significantly associated with mild cognitive impairment in various neuropsychiatric diseases $(13,14)$.

In the present study, it was first investigated whether exposure to sevoflurane at a postnatal age caused later behavioral alterations in Wistar rats. Next, gene profiling was performed in hippocampus samples from Wistar rats with or without sevoflurane exposure during developmental stages. The study also attempted to explore the underlying molecular mechanisms of long-term neural impairment caused by exposure to sevoflurane.

\section{Materials and methods}

Animals. According to previous observations (15), a total fo 49 , male Wistar rats $(14.54 \pm 1.52 \mathrm{~g})$ at postnatal day 7 (P7) were selected for experimental analyses. The Wistar rats at P7 were purchased from the Model Animal Research Center of Nanjing University (Nanjing, China). Rats were housed in polypropylene cages under a 12 -h alternating light/dark cycle, with food and water supplied ad libitum in the institutional animal facilities. All the experimental protocols were approved by the Institutional Animal Care and Use Committee of the First Affiliated Hospital of Bengbu Medical College (Anhui, China) and performed according to the Guide for the Care and Use of Laboratory Animals (16). All efforts were made to minimize animal suffering and to reduce the number of animals used.

Anesthesia methods. A total of $48 \mathrm{~W}$ istar rats at P7 were randomly divided into four groups $(n=12)$, including the 0,2 , 4 and 6-h treatment group, in which Wistar rats were exposed to $3 \%$ sevoflurane for $0,2,4$ and $6 \mathrm{~h}$, respectively. For anesthesia, Wistar rats were placed in a temperature-controlled $\left(37 \pm 0.5^{\circ} \mathrm{C}\right)$ plexiglas anesthesia chamber. First, the rats were subject to $5 \%$ sevoflurane exposure for $30 \mathrm{sec}$, provided in a gas mixture of $5 \%$ carbon dioxide, $21 \%$ oxygen and balanced nitrogen at a flow rate of $10 \mathrm{l} / \mathrm{min}$. Next, the rats were exposed to $3 \%$ sevoflurane for the specified time period at a rate of $1.5 \mathrm{l} / \mathrm{min}$. During the anesthesia process, the concentrations of sevoflurane, carbon dioxide and oxygen in the gas mixture were monitored with an anesthetic gas monitor (Datex-Ohmeda S/5; GE Healthcare Life Sciences, Chicago, IL, USA). Rats were breathing spontaneously during anesthesia. Anesthesia was ended by discontinuing the anesthetics, and then rats were housed in normal conditions until 12 weeks old, at which time behavioral tests were performed.

Behavioral experiments. As described in our previous study (9), three tests were conducted in sequence, including the elevated plus-maze (EPM), O-maze and Y-maze. For each behavioral test, the movement tracks of experimental rats were recorded by a video-tracking software (Any-Maze version 5.1; Stoelting Co., Wood Dale, IL, USA) and analyzed by an additional researcher who was blinded to the experimental protocols. All the test were performed during the dark phase (active period of rats) between 1 a.m. and 4 p.m. The experimental details of EPM test, O-maze and Y-maze were as described in previous studies $(9,17-19)$. Briefly, the EPM and $\mathrm{O}$-maze tests were used to assess the anxiety-like behavior in rodents, while the Y-maze test was used to investigate the immediate spatial working memory (a pattern of manifestation of cognitive function) of rodents.

Gene expression microarray analysis. Rats were anaesthetized by isoflurane with an induction dosage of $4 \%$, maintained at 2\% (RuiTaibio, Beijing, China) and decapitated to obtain the hippocampus, which was then stored at $-80^{\circ} \mathrm{C}$ until RNA extraction. Total RNA was extracted from the hippocampal tissues using a standard TRIzol reagent (catalogue no. 15596026; Thermo Fisher Scientific, Inc., Waltham, MA, USA) as described previously (9). In brief, after the tissue was homogenized, $0.3 \mathrm{ml}$ TRIzol was added to each sample. Then, $0.3 \mathrm{ml} \mathrm{100 \%} \mathrm{chloroform} \mathrm{(Sinopharm}$ Chemical Reagent Co., Ltd, Shanghai, China) was added to stratify the sample solution; transfer the aqueous phase containing the RNA to a new tube. Finally, $0.5 \mathrm{ml}$ isopropanol was added to the aqueous phase to precipitate the total RNA. The quality and concentration of the RNA samples were then assessed at he absorbance ratios of A260/280 and A260/230 using a NanoDrop ND-1000 spectrophotometer (Thermo Fisher Scientific, Inc.), and samples were denatured by $2 \%$ agarose gel electrophoresis.

The whole transcription profile of all mRNAs targeted by the microarray for each sample was determined using an Affymetrix Rat Genome U34 Array (Thermo Fisher Scientific, Inc.). Sample labeling and array hybridization were performed according to the manufacturer's instructions with minor modifications. In order to analyze the gene expression, CapitalBio Corporation (Beijing, China) completed the following steps: Briefly, mRNA was purified from total RNA following the removal of rRNA using mRNA-ONLY ${ }^{\mathrm{TM}}$ Eukaryotic mRNA Isolation kit (Epicentre, Madison, WI, USA). Next, each sample was amplified and transcribed into fluorescent cDNA along the entire length of the transcripts without 3' bias using random primers (catalogue no. 79236; Qiagen, Hilden, Germany). Subsequent to purification with 
an RNeasy Mini kit (Qiagen, Hilden, Germany), the labeled cDNAs were hybridized with the specific probes on the Array. The hybridized arrays were washed, fixed and scanned at $5 \mathrm{~mm}$ /pixel resolutions with an Agilent DNA microarray scanner (G2505C; Agilent Technologies, Inc., Santa Clara, CA, USA).

Upon collection of signal, technical quality control was performed using dChip version 2005 (Affymetrix; Thermo Fisher Scientific, Inc.) with the default settings. Expression data were normalized by quantile normalization and the robust multichip average algorithm, as previously described (20). Probe-level files were generated following normalization. According to the fold change (FC) analysis (FC >2.0) and false discovery rate (FDR) analysis (FDR $<0.05)$, differentially expressed genes were identified through FC filtering according to the predetermined $\mathrm{P}$-value threshold for significant differences (set at $\mathrm{P}<0.05$ ).

Reverse transcription-quantitative polymerase chain reaction $(R T-q P C R)$. Total RNA was reversely transcribed into cDNA using a PrimeScript RT Reagent kit with gDNA Eraser (Takara Biotechnology Co., Ltd., Dalian, China) following the manufacturer's instructions, as previously described (21). Next, qPCR was performed using a SYBR Green PCR kit (Takara Biotechnology Co., Ltd.) on a CFX96 Real-Time PCR Detection System (Bio-Rad Laboratories, Inc., Hercules, CA, USA). The PCR conditions included an initial step at $95^{\circ} \mathrm{C}$ for $5 \mathrm{~min}$, followed by 40 cycles of annealing and extension, prior to quantification at $95^{\circ} \mathrm{C}$ for $15 \mathrm{sec}$ and $60^{\circ} \mathrm{C}$ for $30 \mathrm{sec}$. Each cDNA sample was analyzed in triplicate, in a final volume of $25 \mu \mathrm{l}$, containing $1 \mu \mathrm{l} \mathrm{cDNA}, 400 \mathrm{nM}$ of the forward and reverse gene-specific primers $(1 \mu \mathrm{l}), 12.5 \mu \mathrm{l} 2 \mathrm{x}$ SYBR Green master mix (catalogue no. 639676; Takara Biotechnology Co., Ltd.) and $10.5 \mu \mathrm{l}$ distilled water. The relative gene expression level was quantified based on the cycle threshold values (22) and normalized to the reference gene, which was glyceraldehyde 3-phosphate dehydrogenase (GAPDH). Primer sequences used were listed as follows: KIF2A: F 5'-ATTTTCTCTCATTGA CCTGGCTG-3', R 5'-ACTCCTTGAGTGCTAAAAGGC-3'; $R Y B P:$ F 5'-CGACCAGGCCAAAAAGACAAG-3', R 5'-CAC ATCGCAGATGCTGCAT-3'; DOCK7: F 5'-CCATCTGGA AGCGCCTTTG-3', R 5'-ACGATGATCTCTAGCGTGTCT-3'; CDC40: F 5'-CTCTAGCTGCTTCGTATGGCT-3', R 5'-CAA GTGCATGAGAGAGTCCGC-3'; PTBP3: F 5'-CCAGCC ATTGGATTTCCTCAA-3', R 5'-AAAAAGCCCATGTGG TGTGATA-3'; NRTN: F 5'-GGGCTACACGTCGGATGA G-3', R 5'-CCAGGTCGTAGATGCGGATG-3'; TMEM205: F 5'-CACTTGCTGGTCTTGTCTGGT-3', R 5'-GGAGACGTG AAAATAGACTGGG-3'; SLC39A3: F 5'-GGTGGCGTATTC CTGGCTAC-3', R 5'-CTGCTCCACGAACACAGTGA-3'; CDCA3: F 5'-GAGTAGCAGACCCTCGTTCAC-3', R 5'-TCT CTACCTGAATAGGAGTGCG-3'; KIFlC: F 5'-AGTGTG GGTTTGTGTGTATGAG-3', R 5'-CCAGCATCGCACCAT GTAGA-3'; CASP3: F 5'-ATGGAGAACAACAAAACCTCA GT-3', R 5'-TTGCTCCCATGTATGGTCTTTAC-3'; GAPDH: F 5'-AGGTCGGTGTGAACGGATTTG3', R 5'-TGTAGACCA TGTAGTTGAGGTCA-3'.

Immunohistochemicalassay. Following sacrifice, the entire rat brain was rapidly removed, washed with phosphate-buffered saline, incubated for at least $48 \mathrm{~h}$ in $4 \%$ paraformaldehyde (Sigma-Aldrich; Merck, Darmstadt, Germany) and embedded in paraffin. Next, the paraffin-embedded tissues were sectioned into 4- $\mu \mathrm{m}$ slices, and sections with the hippocampus structure were used for immunohistochemical analyses. Slices were incubated with rabbit anti-caspase-3 primary antibody (Catalogue no. AC030; Beyotime Institute of Biotechnology; $1: 200)$ at $4{ }^{\circ} \mathrm{C}$ overnight, then incubated with biotinylated anti-rabbit secondary antibody (catalogue no. A0277; Beyotime Institute of Biotechnology; 1:1,000) for $30 \mathrm{~min}$ at $37^{\circ} \mathrm{C}$, and immunoreactivity was then visualized by addition of a streptavidin-peroxidase complex and 3,3'-diaminobenzidine (both from Beyotime Institute of Biotechnology). Counterstaining was performed with hematoxylin (Zhongshan Golden Bridge, Beijing, China). Subsequent to each incubation step, slices were washed with Tris-buffered saline/Tween 20 three times for 5 min each. All images were captured using an Axioskop fluorescence microscope (Carl Zeiss AG, Oberkochen, Germany).

Western blotting. The preparation of hippocampal tissues for protein extraction was performed as described in previous studies $(23,24)$. Briefly, total proteins were extracted using the radioimmunoprecipitation assay buffer (Beyotime Institute of Biotechnology). The hippocampus tissue proteins were separated by $10 \%$ sodium dodecyl sulfate-polyacrylamide gel electrophoresis and then electrotransferred to a nitrocellulose membrane. After blocking with 5\% non-fat milk for $1 \mathrm{~h}$ at room temperature and being washed three times (10 min each) using $1 \mathrm{x}$ TBST, the membrane was incubated with primary antibodies at $4^{\circ} \mathrm{C}$ overnight and then with horseradish peroxidase-conjugated secondary antibody (Beyotime Institute of Biotechnology) at room temperature for $2 \mathrm{~h}$. The primary antibodies used were as follows: Rabbit anti-cleaved-poly (ADP-ribose) polymerase (PARP) antibody (catalogue no. AP102; 1:200) and rat anti-GAPDH antibody (catalogue no. AG019; 1:5,000; both from Beyotime Institute of Biotechnology). Subsequently, the target proteins were visualized by an enhanced chemiluminescence method (catalogue no. W1001, Promega Corporation, Madison, WI, USA) and analyzed with the Gel Image Documentation System (Wealtec Corp., Sparks, NV, USA). The relative level of PARP was normalized to that of GAPDH, as presented by band intensity.

Statistical analysis. All data are presented as the mean \pm standard error of the mean, and all statistical analyses were conducted using GraphPad Prism (version 5.0; GraphPad Software, Inc., La Jolla, CA, USA) and SPSS (version 17.0; SPSS, Inc., Chicago, IL, USA) software. For behavioral tests and RT-qPCR results, one-way analysis of variance (ANOVA) was applied to compare intergroup differences with Bonferroni post hoc tests. The correlation analysis was performed using Pearson's correlation coefficients. For western blotting and immunohistochemistry results, two-tailed Student's t-test was applied for comparison. A P-value of $<0.05$ was considered to indicate differences that were statistically significant. Any additional experimental data not provided in the current study are indicated by 'data not shown' and can be obtained upon request. 


\section{Results}

Sevoflurane exposure at P7 causes long-term spatial working memory impairment in adult rats. In order to investigate the long-term effect of sevoflurane exposure on cognitive functions, the infant rats at $\mathrm{P} 7$ were exposed to $3 \%$ sevoflurane for different time durations $(0,2,4$ or $6 \mathrm{~h})$, and behavioral tests were then performed when the rats were 12-weeks-old. As shown in Fig. 1A, there was no significant difference in the exploration time, manifested as the time spent in the open arms of the maze, in the EPM test between rats receiving 3\% sevoflurane exposure and those without exposure (one-way ANOVA, P $>0.05$ for each comparison with the $0 \mathrm{~h}$ group). Similarly, there was also no difference in the total activity, which was demonstrated by the total number of arm entries, among groups receiving 3\% sevoflurane exposure for different time lengths (one-way ANOVA, P>0.05 vs. the 0 h group; Fig. 1B). Furthermore, in the $\mathrm{O}$-maze test, no difference was observed in the latency to enter the anxiety-associated bright compartment and in the total time spent in the open sector (one-way ANOVA, P>0.05; Fig. $1 \mathrm{C}$ and D).

As for the Y-maze test, in which rats had to select between two lanes when facing the Y-shaped track, rats receiving 3\% sevoflurane exposure during the early postnatal stage for 4 and $6 \mathrm{~h}$ exhibited significantly reduced spontaneous alterations in arm entries when compared with those without sevoflurane exposure (Fig. 1E; one-way ANOVA, P<0.05). By contrast, there was no significant difference between rats with $3 \%$ sevoflurane exposure for $2 \mathrm{~h}$ and control rats with 0 -h exposure $(\mathrm{P}=0.594)$. In addition, it was observed that longer time of sevoflurane exposure may induce more severe spatial working memory impairment, but the difference was not statistically significant ( $6 \mathrm{~h}$ vs. $4 \mathrm{~h}$ groups). However, the number of arm entries presented no significant difference among groups, suggesting that the spatial working memory impairment may not be due to weakened movements ( $\mathrm{P}>0.05$ vs. the $0 \mathrm{~h}$ group; Fig. 1F).

Gene profiling is dysregulated in the hippocampus of adult rats exposed to sevoflurane at $P 7$. As shown in Fig. 1E, exposure to $3 \%$ sevoflurane for $6 \mathrm{~h}$ at P7 caused the most severe spatial working memory impairment in aged rats; therefore, rats from this group were selected for the following analyses. In order to explore the molecular pathways underlying the sevoflurane-induced long-term spatial working memory impairment, transcriptome analysis of the hippocampus, the major structure participating in the regulation of cognitive functions, was first performed in adult rats from the 6-h group $(n=3)$. Meanwhile, normal rats $(n=3)$ that did not receive $3 \%$ sevoflurane exposure were used as the controls.

A total of 7,127 probes were detected, among which 264 genes demonstrated significantly different expression levels between rats from the $6 \mathrm{~h}$ group and the controls (fold change $>2.0, \mathrm{P}<0.05$ and FDR $<0.05$; data not shown). Among the dysregulated 264 genes, 51 were downregulated and 213 were upregulated. In order to validate the accuracy of mRNA array, a total of 10 dysregulated mRNAs, including 5 upregulated genes, namely KIF2A, RYBP, DOCK7, CDC40 and PTBP3, and 5 downregulated genes, namely NRTN, TMEM205, SLC39A3, CDCA3 and KIF1C, were randomly selected for
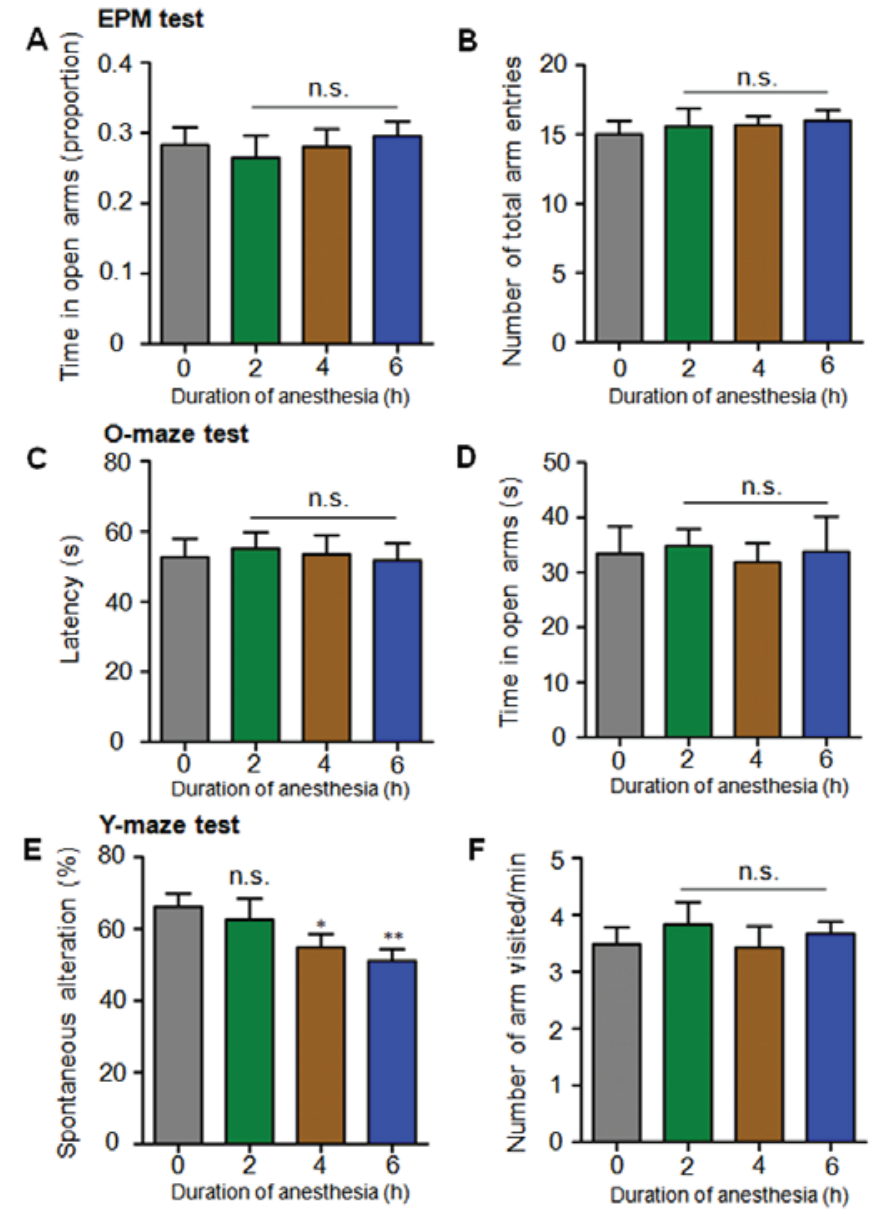

Figure 1. Behavioral tests of adult rats exposed to $3 \%$ sevoflurane for 0,2 , 4 and $6 \mathrm{~h}$ at postnatal day 7. (A) The time spent in the open arms of the maze (proportion) and (B) number of total arm entries were investigated by the EPM test. (C) The latency to enter the open arms (sec) and (D) time spent in the open arms (sec) were examined in O-maze test. (E) Spontaneous alternations $(\%)$, and $(\mathrm{F})$ number of arms visited per minute were analyzed by Y-maze test. No significant differences were observed in the EPM and $\mathrm{O}$-maze tests between rats with and without $3 \%$ sevoflurane exposure. In the Y-maze test, exposure to $3 \%$ sevoflurane for 4 and $6 \mathrm{~h}$ led to decreased spontaneous alternations, but did not disturb the number of arms visited $/ \mathrm{min}$. Data are expressed as the mean \pm standard deviation $(n=12$ per group; one-way analysis of variance and Bonferroni's post hoc test). ${ }^{*} \mathrm{P}<0.05$ and ${ }^{* *} \mathrm{P}<0.01$ vs. the $0 \mathrm{~h}$ group. n.s., not significant difference with $0 \mathrm{~h}$ group; EPM, elevated plus-maze.

further verification using RT-qPCR. All the 10 selected genes presented consistent expression levels in terms of the regulatory direction with the results extracted from mRNA array (data not shown).

Increased caspase-3 gene (CASP3) expression is correlated with long-term spatial working memory performance of rats exposed to sevoflurane at P7. Specifically, the expression of CASP3 (encoding caspase-3 protein) was observed to be the most significantly upregulated gene from microarray data obtained in the current study $(\mathrm{FC}=2.53)$. This gene has previously been implicated in the impairment due to exposure to $(25,26)$. Therefore, the current study selected this gene for subsequent analysis. Firstly, RT-qPCR was performed to validate whether CASP3 was indeed upregulated in the hippocampus of experimental rats when compared with healthy rats. As shown in Fig. 2A, CASP3 expression level 

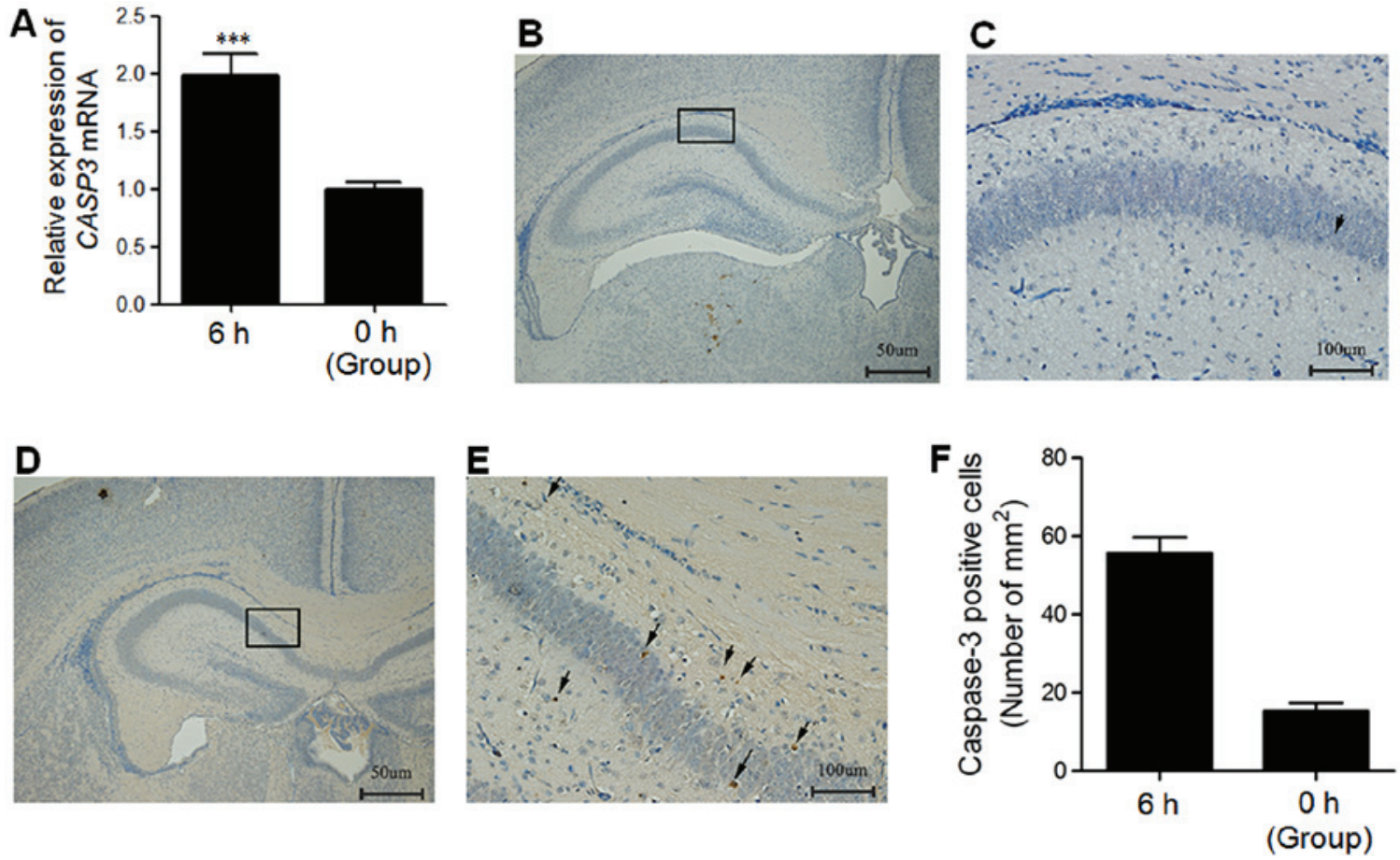

Figure 2. Expression of caspase-3 was upregulated in the hippocampus of adult rats exposed to $3 \%$ sevoflurane for $6 \mathrm{~h}$ at P7 compared with control rats without exposure. (A) Quantitative polymerase chain reaction of CASP3 expression in the hippocampus of adult rats. Immunohistochemical staining of caspase-3 in the hippocampus of adult rats without $3 \%$ sevoflurane exposure is shown at (B) $x 4$ and (C) x20 magnification. Immunohistochemical staining of caspase-3 in the hippocampus of adult rats exposed to $3 \%$ sevoflurane for $6 \mathrm{~h}$ at postnatal day 7 is demonstrated at (D) $\mathrm{x} 4$ and (E) x20 magnification. Scale bar, 50 (at $\mathrm{x} 4$ magnification) or $100 \mu \mathrm{m}$ (at x 20 magnification). Caspase-3 positive cells were indicated by arrows. (F) Statistical analysis of caspase-3 positive cells in the hippocampus of adult rats. Data are expressed as the mean \pm standard error ( $\mathrm{n}=3$ per group; two-tailed Student's t-test). ${ }^{* * *} \mathrm{P}<0.001 \mathrm{vs}$. control $(0 \mathrm{~h})$ group. CASP3, caspase-3 gene.

in the hippocampus of rats with exposure to sevoflurane was 1.97 times higher than those without sevoflurane exposure $(\mathrm{P}<0.001)$. Immunohistochemical analysis of the hippocampal tissue sections also validated this observation. As shown in Fig. 2B-F, the number of caspase-3 positive cells in the hippocampus of experimental rats was approximately 4 times higher in comparison with those in healthy rats $(\mathrm{P}<0.001)$.

Since the spatial working memory was impaired more severely in rats exposed to sevoflurane for $6 \mathrm{~h}$ as compared with rats exposed for 2 and $4 \mathrm{~h}$, or compared with the control group, it was speculated that the alteration of spatial working memory performance may partly be due to the fluctuated CASP3 expression. Supporting our hypothesis, further analysis revealed that the CASP3 mRNA level was negatively correlated with the spatial working memory in experimental rats $(n=36$; $\mathrm{r}^{2}=0.279, \mathrm{P}=0.043$; Fig. 3). Insufficient negative association between CASP3 mRNA level and spatial working memory performance in experimental rats suggested that genes than other CASP3 may also be involved in the dysregulation of spatial working memory impairment.

Cleaved-PARP level is increased in the hippocampus of adult rats exposed to sevoflurane at P7. As a substrate of caspase-3, PARP has been demonstrated to also be associated with long-term memory impairment. Therefore, the present study examined whether PARP was cleaved by caspase-3 in experimental rats by western blotting. As shown in Fig. 4, the relative level of cleaved-PARP was significantly

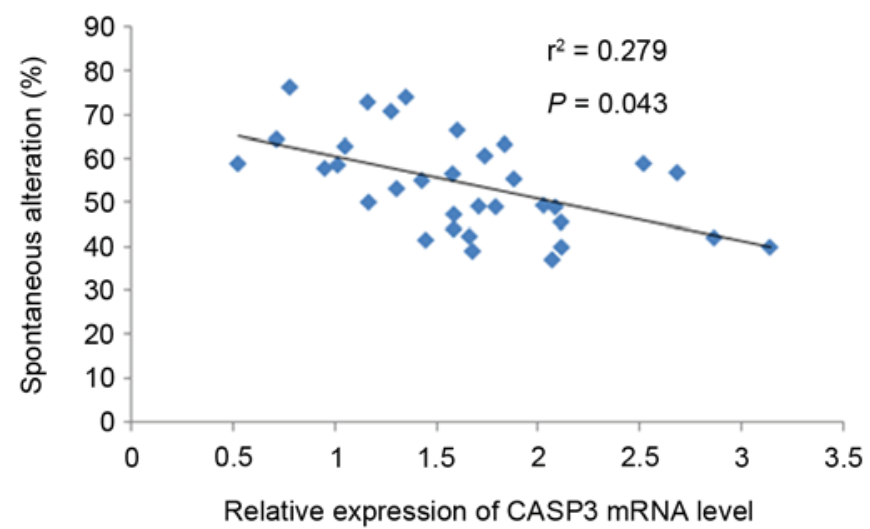

Figure 3. $C A S P 3$ expression was negatively correlated with spatial working memory in adult rats with exposure to $3 \%$ sevoflurane at postnatal day 7 . $C A S P 3$, caspase-3 gene.

increased in adult rats with exposure to sevoflurane for $6 \mathrm{~h}$ at P7, when compared with those without sevoflurane exposure $(P=0.002)$, further confirming that the caspase-3/PARP pathway may be involved in long-term memory impairment.

\section{Discussion}

In the present study, the long-term impairment effect on cognitive functions of sevoflurane exposure at the postnatal stage was validated through in vivo animal experiments, which is 
A

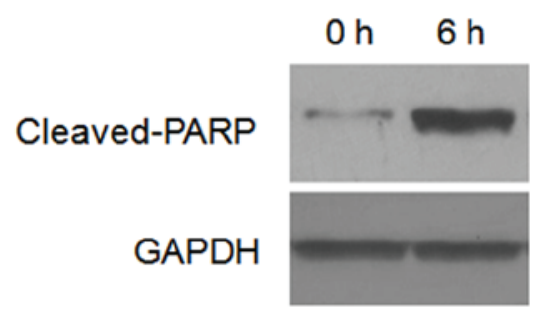

B

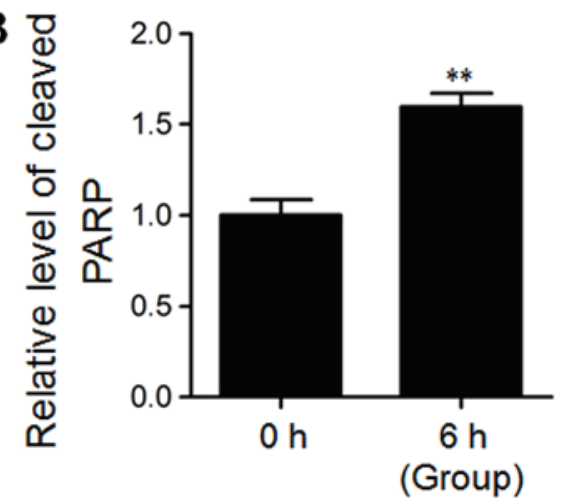

Figure 4. Protein level of cleaved-PARP was upregulated in the hippocampus of rats receiving $3 \%$ sevoflurane at postnatal day 7 compared with the control rats. (A) Images of western blotting; (B) statistical analysis of the relative level of cleaved-PARP in each group. ${ }^{* *} \mathrm{P}<0.01 \mathrm{vs}$. control ( 0 h) group (two-tailed Student's t-test). PARP, poly (ADP-ribose) polymerase; P7, postnatal day 7.

in accordance to the observations of previous studies $(6,15)$. In particular, significant spatial working memory impairment was detected in adult rats exposed to $3 \%$ sevoflurane for $6 \mathrm{~h}$ at P7, while rats receiving 3\% sevoflurane exposure for $2 \mathrm{~h}$ or without exposure did not present impairment. However, the underlying molecular pathways of the long-term cognitive impairment induced by $3 \%$ sevoflurane exposure in postnatal rats remain unclear.

Supporting the results of the present study, several previous studies have indicated that exposure to sevoflurane for $6 \mathrm{~h}$ significantly upregulated the expression of caspase- 3 in the hippocampus of postnatal rats (27), while upregulation of caspase-3 may lead to memory and cognitive impairment though mediating neural apoptosis in the hippocampus. As a substrate of caspase-3, PARP can be cleaved by caspase-3, and increased cleaved-PARP levels have been observed to be associated with long-term memory impairment (28). As such, the expression data of caspase-3 and PARP suggest that apoptosis serves an important role in the cognitive impairment caused by exposure to $3 \%$ sevoflurane in postnatal rats. Apoptosis is a genetically controlled mechanism of cell death involved in the regulation of tissue homeostasis. Typically, B-cell lymphoma-2 (Bcl-2) and caspase-3 are the main factors inhibiting apoptosis and the final executive protein in apoptosis, respectively, while $\mathrm{Bcl}-2$-associated $\mathrm{X}$ protein may induce apoptosis by increasing the mitochondrial membrane permeability (29). However, other molecular pathways also underlie the neurological damage caused by exposure to anesthetics. One such example is the Rho/Rho-kinase pathway. According to the study by Lemkuil et al (30), isoflurane induced neurotoxicity in rats by activating $\mathrm{p} 75^{\mathrm{NTR}}$ - RhoA, while inhibition of the activity of RhoA reversed the neurotoxicity of isoflurane. In addition, Pearn et al (31) reported that $\mathrm{p} 75^{\mathrm{NTR}}$ and RhoA kinase activation were involved in propofol-induced apoptosis in developing neurons in vitro and in vivo. The present study added novel results regarding the roles of caspase- 3 and cleaved-PARP in sevoflurane exposure-induced cognitive impairment, and highlighted the importance of caspase- 3 and cleaved PARP in the pathogenesis of nervous system dysfunction.

Another interesting phenomenon observed in the current study is that $3 \%$ sevoflurane exposure for $>4 \mathrm{~h}$ at $\mathrm{P} 7$ was able to impair the cognitive functions of adult rats, while $3 \%$ sevoflurane exposure for $<2 \mathrm{~h}$ did not have an evident effect. Consistent with our results, Han et al (15) reported that exposure to $2 \%$ sevoflurane at postnatal days 7-9 ( $2 \mathrm{~h}$ per day) may induce significantly impaired cognitive function in rats reaching adulthood, whereas receiving $2 \%$ sevoflurane for $2 \mathrm{~h}$ for only 1 day (at P7) did not result in impaired cognitive function in adult rats. Shen et al (32) also identified that sevoflurane exerted a dose-dependent neurotoxicity on the neurons. More specifically, rats in the developmental stages (P7) did not present cognitive impairment and nerve inflammation due to exposure to $3 \%$ sevoflurane for $2 \mathrm{~h}$ at one time, while those inhaling $3 \%$ sevoflurane for $2 \mathrm{~h}$ per day for 3 consecutive days exhibited marked impairment of cognition. Furthermore, Peng et al (33) revealed that low concentration of sevoflurane (1.5\%) may not cause significant cognitive impairment even with exposure for as long as 3 days ( $2 \mathrm{~h}$ per day), while high concentration of sevoflurane $(3 \%)$ for only $2 \mathrm{~h}$ was sufficient to induce severe cognitive impairment. Therefore, the present study, along with other similar studies, suggested that minimizing the use dosage and concentration of anesthetics, particularly sevoflurane, in clinical practice may greatly reduce the neurological damage.

Several limitations in the current study need to be acknowledged. Firstly, due to limitation of financial support, gene profiling analyses were only performed on rats from the $6 \mathrm{~h}$ experimental and control groups, but not on those receiving sevoflurane exposure for 2 or $4 \mathrm{~h}$. Since the degree of cognitive impairment varies among different experimental groups, it may be meaningful to analyze the difference of gene profiling among groups. In addition, the apoptosis of neurons from the hippocampus of experimental and control groups was not detected. Since caspase-3 upregulation has been widely reported to be associated with apoptosis, the apoptosis of neurons from the hippocampus would be more severe in experimental groups, and even exacerbated along with the time of exposure to $3 \%$ sevoflurane; however, this requires further investigation.

In conclusion, the present study identified multiple genes, which may be involved in long-term cognitive impairment caused by exposure to sevoflurane. The upregulation of caspase-3/cleaved-PARP specifically may reveal a novel mechanism, which may be useful for the clinical prevention of cognitive impairment. 


\section{Acknowledgements}

The current study was supported by the Anhui Education Department (grant no. KJ2015B004by) and the Bengbu Medical College Innovation Grant (grant no. BYKY1424ZD).

\section{References}

1. Liang G, Ward C, Peng J, Zhao Y, Huang B and Wei H: Isoflurane causes greater neurodegeneration than an equivalent exposure of sevoflurane in the developing brain of neonatal mice. Anesthesiology 112: 1325-1334, 2010.

2. Xiao H, Liu B, Chen Y and Zhang J: Learning, memory and synaptic plasticity in hippocampus in rats exposed to sevoflurane. Int J Dev Neurosci 48: 38-49, 2016.

3. Satomoto M, Satoh Y, Terui K, Miyao H, Takishima K, Ito M and Imaki J: Neonatal exposure to sevoflurane induces abnormal social behaviors and deficits in fear conditioning in mice. Anesthesiology 110: 628-637, 2009.

4. Erden V, Erkalp K, Yangin Z, Delatioglu H, Kiroglu S, Ortaküz S and Ozdemir B: The effect of labor on sevoflurane requirements during cesarean delivery. Int J Obstet Anesth 20: 17-21, 2011.

5. Lopéz Gil ML, Brimacombe J and Clar B: Sevoflurane versus propofol for induction and maintenance of anaesthesia with the laryngeal mask airway in children. Paediatr Anaesth 9: 485-490, 1999.

6. Kalkman CJ, Peelen L, Moons KG, Veenhuizen M, Bruens M, Sinnema G and de Jong TP: Behavior and development in children and age at the time of first anesthetic exposure. Anesthesiology 110: 805-812, 2009.

7. Wilder RT, Flick RP, Sprung J, Katusic SK, Barbaresi WJ, Mickelson C, Gleich SJ, Schroeder DR, Weaver AL and Warner DO: Early exposure to anesthesia and learning disabilities in a population-based birth cohort. Anesthesiology 110: 796-804, 2009.

8. Zhang DX, Jiang S, Yu LN, Zhang FJ, Zhuang Q and Yan M: The effect of sevoflurane on the cognitive function of rats and its association with the inhibition of synaptic transmission. Int J Clin Exp Med 8: 20853-20860, 2015.

9. Ling YZ, Ma W, Yu L, Zhang Y and Liang QS: Decreased PSD95 expression in medial prefrontal cortex (mPFC) was associated with cognitive impairment induced by sevoflurane anesthesia. J Zhejiang Univ Sci B 16: 763-771, 2015.

10. Xiong WX,Zhou GX, Wang B, Xue ZG, Wang L, Sun HC and Ge SJ: Impaired spatial learning and memory after sevoflurane-nitrous oxide anesthesia in aged rats is associated with down-regulated cAMP/CREB signaling. PLoS One 8: e79408, 2013.

11. Dawe RJ, Bennett DA, Schneider JA and Arfanakis K Neuropathologic correlates of hippocampal atrophy in the elderly: A clinical, pathologic, postmortem MRI study. PLoS One 6: e26286, 2011.

12. Deng M, Hofacer RD, Jiang C, Joseph B, Hughes EA, Jia B, Danzer SC and Loepke AW: Brain regional vulnerability to anaesthesia-induced neuroapoptosis shifts with age at exposure and extends into adulthood for some regions. $\mathrm{Br} \mathbf{J}$ Anaesth 113: 443-451, 2014.

13. Apostolova LG, Green AE, Babakchanian S, Hwang KS, Chou YY, Toga AW and Thompson PM: Hippocampal atrophy and ventricular enlargement in normal aging, mild cognitive impairment (MCI), and Alzheimer Disease. Alzheimer Dis Assoc Disord 26: 17-27, 2012.

14. Zhang J, Yu C, Jiang G, Liu W and Tong L: 3D texture analysis on MRI images of Alzheimer's disease. Brain Imaging Behav 6: 61-69, 2012.

15. Han T,Hu Z, Tang Y, Shrestha A, Ouyang W and Liao Q: Inhibiting Rho kinase 2 reduces memory dysfunction in adult rats exposed to sevoflurane at postnatal days 7-9. Biomed Rep 3: 361-364, 2015.
16. Barthold SW, Bayne K and Davis M: Washington: National Academy Press, 2011.

17. Zhang W, Hetzel A, Shah B, Atchley D, Blume SR, Padival MA and Rosenkranz JA: Greater physiological and behavioral effects of interrupted stress pattern compared to daily restraint stress in rats. PLoS One 9: e102247, 2014.

18. Xie K, Kuang H and Tsien JZ: Mild blast events alter anxiety, memory, and neural activity patterns in the anterior cingulate cortex. PLoS One 8: e64907, 2013.

19. Heo H, Shin Y, Cho W, Choi Y, Kim H and Kwon YK: Memory improvement in ibotenic acid induced model rats by extracts of Scutellaria baicalensis. J Ethnopharmacol 122: 20-27, 2009.

20. López-Romero P, González MA, Callejas S, Dopazo A and Irizarry RA: Processing of Agilent microRNA array data. BMC Res Notes 3: 18, 2010.

21. Li X, Yang Q, Bai J, Yang Y, Zhong L and Wang Y: Identification of optimal reference genes for quantitative PCR studies on human mesenchymal stem cells. Mol Med Rep 11: 1304-1311, 2015.

22. Livak KJ and Schmittgen TD: Analysis of relative gene expression data using real-time quantitative PCR and the 2(-Delta Delta C(T)) Method. Methods 25: 402-408, 2001.

23. BradleyEW,CarpioLR,McGee-LawrenceME,CastillejoBecerraC, Amanatullah DF, Ta LE, Otero M, Goldring MB, Kakar S and Westendorf JJ: Phlppl facilitates post-traumatic osteoarthritis and is induced by inflammation and promoter demethylation in human osteoarthritis. Osteoarthritis Cartilage 24: 1021-1028, 2016

24. Lin D and Zuo Z: Isoflurane induces hippocampal cell injury and cognitive impairments in adult rats. Neuropharmacology 61: 1354-1359, 2011.

25. Deng B, Xu H, Zhang J, Wang J, Han LC, Li LY, Wu GL, Hou YN, Guo GZ, Wang Q, et al: Neuroprotective effects of sevoflurane against electromagnetic pulse-induced brain injury through inhibition of neuronal oxidative stress and apoptosis. PLoS One 9: e91019, 2014.

26. Fang F, Lin W, Ling X, Song R, Liu Q, Lai B and Cang J: The hippocampal cyclin D1 expression is involved in postoperative cognitive dysfunction after sevoflurane exposure in aged mice. Life Sci 160: 34-40, 2016.

27. Zhou X, Song FH, He W, Yang XY, Zhou ZB, Feng X and Zhou LH: Neonatal exposure to sevoflurane causes apoptosis and reduces nNOS protein expression in rat hippocampus. Mol Med Rep 6: 543-546, 2012.

28. Cohen-Armon M, Visochek L, Katzoff A, Levitan D, Susswein AJ, Klein R, Valbrun M and Schwartz JH: Long-term memory requires polyADP-ribosylation. Science 304: 1820-1822, 2004.

29. Negrín G, Rubio S, Marrero MT, Quintana J, Eiroa JL, Triana J and Estévez F: The eudesmanolide tanapsin from Tanacetum oshanahanii and its acetate induce cell death in human tumor cells through a mechanism dependent on reactive oxygen species. Phytomedicine 22: 385-393, 2015.

30. Lemkuil BP, Head BP, Pearn ML, Patel HH, Drummond JC and Patel PM: Isoflurane neurotoxicity is mediated by p75NTR-R hoA activation and actin depolymerization. Anesthesiology 114: 49-57, 2011.

31. Pearn ML, Hu Y, Niesman IR, Patel HH, Drummond JC, Roth DM, Akassoglou K, Patel PM and Head BP: Propofol neurotoxicity is mediated by $\mathrm{p} 75$ neurotrophin receptor activation. Anesthesiology 116: 352-361, 2012.

32. Shen X, Dong Y, Xu Z, Wang H, Miao C, Soriano SG, Sun D, Baxter MG, Zhang Y and Xie Z: Selective anesthesia-induced neuroinflammation in developing mouse brain and cognitive impairment. Anesthesiology 118: 502-515, 2013.

33. Peng S, Zhang Y, Sun DP, Zhang DX, Fang Q and Li GJ: The effect of sevoflurane anesthesia on cognitive function and the expression of Insulin-like Growth Factor-1 in CA1 region of hippocampus in old rats. Mol Biol Rep 38: 1195-1199, 2011. 